\title{
Le déficit en tyrosine kinase ZAP-70: un modèle de déficit immunitaire héréditaire pour l'analyse de l'activation et de la différenciation des lymphocytes T
}

L'identification des bases moléculaires de certains déficits immunitaires héréditaires rares a permis de mettre en évidence le rôle de certaines protéines dans le système immunitaire [1]. Ainsi, à nouveau trois articles récents démontrent que des mutations du gène codant pour la tyrosine kinase ZAP-70 sont à l'origine d'une inhibition presque totale des fonctions immunes des lymphocytes T ( $m / s n^{\circ} 10$, vol. 10, p. 1038 [2-4]). Ces études sur des patients atteints de déficits immunitaires ont mis en évidence, d'une part, le rôle clé de ZAP-70 dans le développement et l'activation des lymphocytes $\mathrm{T}$ et, d'autre part, le mécanisme moléculaire impliqué dans une forme de déficit immunitaire de transmission autosomique récessive.

Les lymphocytes $\mathrm{T}$ jouent un rôle crucial lors de la réponse immune : les cellules $\mathrm{T}$ cytotoxiques $\mathrm{CD} 8^{+}$tuent les cellules infectées par des virus, les lymphocytes $\mathrm{T}$ helper $\mathrm{CD}^{+}$facilitent la réponse humorale des lymphocytes $B$ et sécrètent des cytokines activatrices des fonctions phagocytaires. Au cours d'une réponse immune, la reconnaissance de l'antigène et l'activation des lymphocytes $\mathrm{T}$ se fait grâce au complexe récepteur $\mathrm{T}$ pour l'antigène (TCR)/CD3 [5]. Le TCR est composé de plusieurs sous-unités: les chaînes $\alpha$ et $\beta$ qui se lient à l'antigène. Il s'y associe le complexe CD3 qui comprend les chaînes $\gamma, \delta, \varepsilon$ ainsi qu'un homodimère $\zeta$, ou un hétérodimère contenant la chaîne $\zeta$, et, soit la chaîne $\eta$, soit la chaîne $\gamma$ du récepteur FcERI. L'importance de ces différentes chaînes associées au TCR

par l'étude de deux déficits immunitaires liés à un défaut d'expression soit de la chaîne $\varepsilon$ [6], soit de la chaîne $\gamma[7]$.

La stimulation du récepteur $\mathrm{T}$ par des peptides antigéniques liés aux molécules du complexe majeur d'histocompatibilité et présentés à la surface de cellules présentatrices de l'antigène induit une cascade d'événements biochimiques conduisant à la différenciation et à la prolifération des cellules $T$. L'activation de tyrosine kinases et la phosphorylation de protéines cellulaires sur des résidus tyrosine est le premier de ces événements [8,9]. Pour l'instant, trois tyrosine kinases ont été impliquées dans la signalisation par le CD3/TCR, Lck, Fyn et ZAP-70 (figure 1).

Lck est une tyrosine kinase de la famille Src de $56 \mathrm{kDa}$ [10], exprimée majoritairement dans les lymphocytes T et associée aux molécules CD4 dans la population helper et aux molécules CD8 dans la population cytotoxique. Un défaut d'expression de Lck, dans des souris obtenues par recombinaison homologue du gène codant pour Lck, induit une forte diminution du nombre de thymocytes et de lymphocytes T circulants $\left(m / s n^{\circ} 5\right.$, vol. $8, p$. 489) [11]. Les quelques lymphocytes $\mathrm{T}$ mûrs restants présentent un défaut de stimulation par le complexe CD3/TCR. Fyn appartient également à la famille Src; cette kinase a un poids moléculaire de $59 \mathrm{kDa}$ et est associée au complexe CD3. L'analyse de souris mutées sur le gène fyn a montré que cette tyrosine kinase est nécessaire à l'activation par le complexe CD3/TCR d'une sous-popula- tion de thymocytes; en revanche, elle ne semble pas indispensable à l'activation des lymphocytes $T$ mûrs [12, 13].

La protéine ZAP-70 a un poids moléculaire apparent de $70 \mathrm{kDa}$ et appartient à la même famille de tyrosine kinases que Syk; elle est exprimée exclusivement dans les cellules $\mathrm{T}$ et les cellules natural killer (NK) [14]. Cette tyrosine kinase a une structure différente de celle des membres de la famille Src. L'absence de myristylation rend sa localisation de façon constitutive à la membrane plasmique peu probable; de plus, elle porte, comme les autres membres de la famille Syk, deux domaines $\mathrm{SH} 2$ (Src homology domain 2) qui jouent un rôle dans l'association avec des protéines phosphorylées sur des résidus tyrosine [15]. Contrairement aux membres de la famille Src, elle ne possède pas de domaine SH3 ni de tyrosine carboxy-terminale régulatrice. Dans les lymphocytes $T$ quiescents, la protéine ZAP-70 n'est pas associée au complexe CD3/TCR mais, après activation, elle s'associe rapidement aux homodimères de chaînes $\zeta$ (d'où son nom, $\zeta$ associated protein-70) et au complexe CD3 [16, 17]. Cette association nécessite à la fois la phosphorylation de résidus tyrosine localisés au niveau de motifs peptidiques des chaînes $\zeta$ dont la séquence est Tyr-XX-Leu-X(6-8)-Tyr-XX-Leu, où X est un acide aminé quelconque (motifs ARAM pour antigen recognition activation motif), et la présence des deux sites SH2 sur ZAP-70. En revanche, l'activité kinase de ZAP-70 n'est pas nécessaire à son association avec $\zeta[18]$. 
L'activation des lymphocytes T nécessite l'activation séquentielle de différentes tyrosine kinases. L'événement initial semble être la stimulation de la $556^{\text {lck }}$, qui induirait la phosphorylation de résidus tyrosine appartenant à des motifs ARAM présents sur la chaîne $\zeta$ et les chaînes $\gamma, \delta, \varepsilon$ du complexe CD3 [18]. Cette phosphorylation permettrait alors l'association des différentes chaînes aux domaines SH2 de ZAP70 qui provoquerait alors une cascade d'événements biochimiques conduisant à l'activation des lymphocytes T (figure 1).

Les observations faites sur des cellules de malades présentant un déficit en ZAP-70 semblent confirmer ce modèle. L'étude de familles de malades a permis l'identification des bases génétiques de ce qui peut être considéré comme une nouvelle forme de déficit immunitaire combiné sévère (DICS) (figure 2). Chez trois malades appartenant à deux familles différentes, une mutation ponctuelle homozygote du gène codant pour ZAP-70 conduit à l'absence de protéine. Cette mutation intronique, située 9 paires de bases (pb) en amont du site accepteur d'un exon, a pour conséquence la création d'un nouveau site d'épissage, 9 pb en amont du site normal, et l'addition de trois acides aminés dans le site catalytique de l'enzyme [2, 3]. L'expression de l'ARNm codant pour cette protéine mutée est normale. Toutefois, des cellules transfectées avec cette forme mutée du gène expriment de façon très réduite $\mathrm{ZAP}-70$, que l'addition de trois acides aminés semble rendre beaucoup plus sensible à la protéolyse. Dans une autre famille, l'absence de ZAP-70 semble être le résultat de deux mutations indépendantes [4]. L'une de ces mutations est identique à celle rapportée plus haut, l'autre a pour résultat le remplacement d'une Ser par une Arg dans le site catalytique de la kinase. Dans la dernière famille, une délétion homozygote de $13 \mathrm{pb}$ induit un décalage du cadre de lecture qui se traduit par une protéine mutante à laquelle il manque 82 acides aminés. Il est intéressant de noter que toutes ces familles appartiennent à la communauté mennonite (secte anabaptiste présente aux États-Unis).

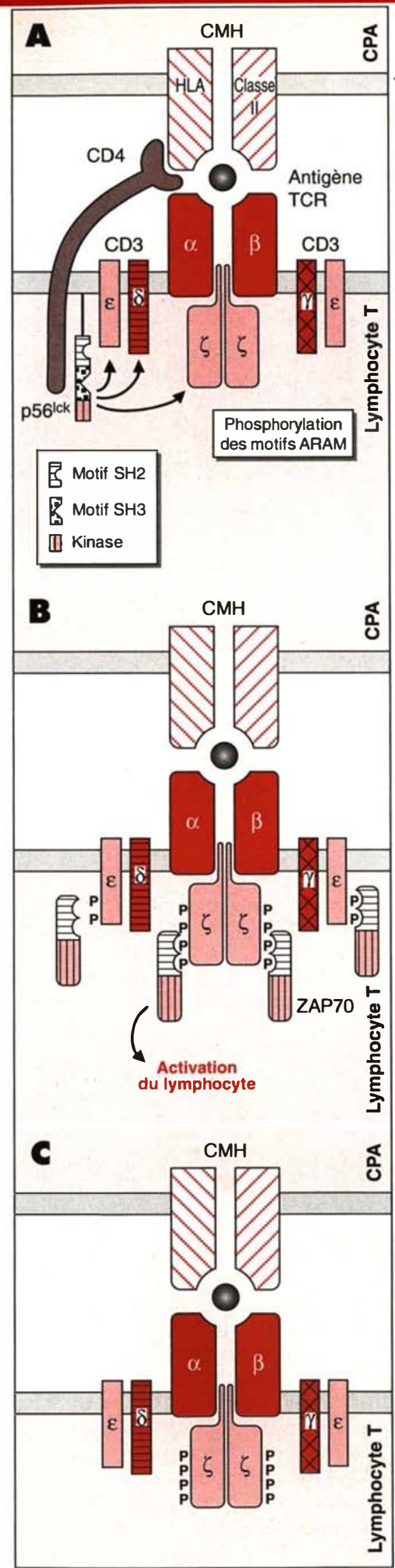

4Figure 1. Événements précoces de I'activation des lymphocytes $T$ par reconnaissance de l'antigène. Dans les cellules normales, la stimulation de TCR active la kinase $p 56^{1 c k}$, provoquant la phosphorylation sur résidus tyrosine de $\mathrm{CD} 3$ et des chaînes $\zeta$ Imotifs ARAM (séquence Tyr-XXLeu-X(6-8)-Tyr-XX-Leu, où $X$ est un acide aminé quelconque)l. ZAP-70 est alors recrutée à la membrane et $s^{\prime}$ associe, par ses sites SH2, au CD3 et au dimère $\zeta$. L'activation séquentielle des différentes kinases entraîne l'activation des lymphocytes T.A. Phosphorylation des motifs ARAM lors de la reconnaissance de l'antigène. B. Recrutement de ZAP-70 et activation Iymphocytaire. C. En I'absence de ZAP-70, donc de son recrutement au CD3, il n'y a pas d'activation du lymphocyte T. CPA : cellule présentatrice de l'antigène $\mathrm{CMH}$ : complexe majeur d'histocompatibilité.

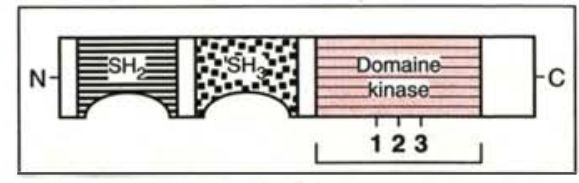

Figure 2. Structure de ZAP-70 et mutations décrites. Les sites de mutations chez les malades atteints de déficit immunitaire sont indiqués en 1, 2, $3:$ (1) terminaison prématurée à la position 503 provoquée par une délétion de $13 \mathrm{pb}$ [4] ; (2) mutation ponctuelle Ser ${ }^{518} \rightarrow$ Arg [3] ; (3) insertion d'un tripeptide Leu-Glu-GIn provoquée par une mutation ponctuelle créant un nouveau site d'épissage $[2,3]$. 


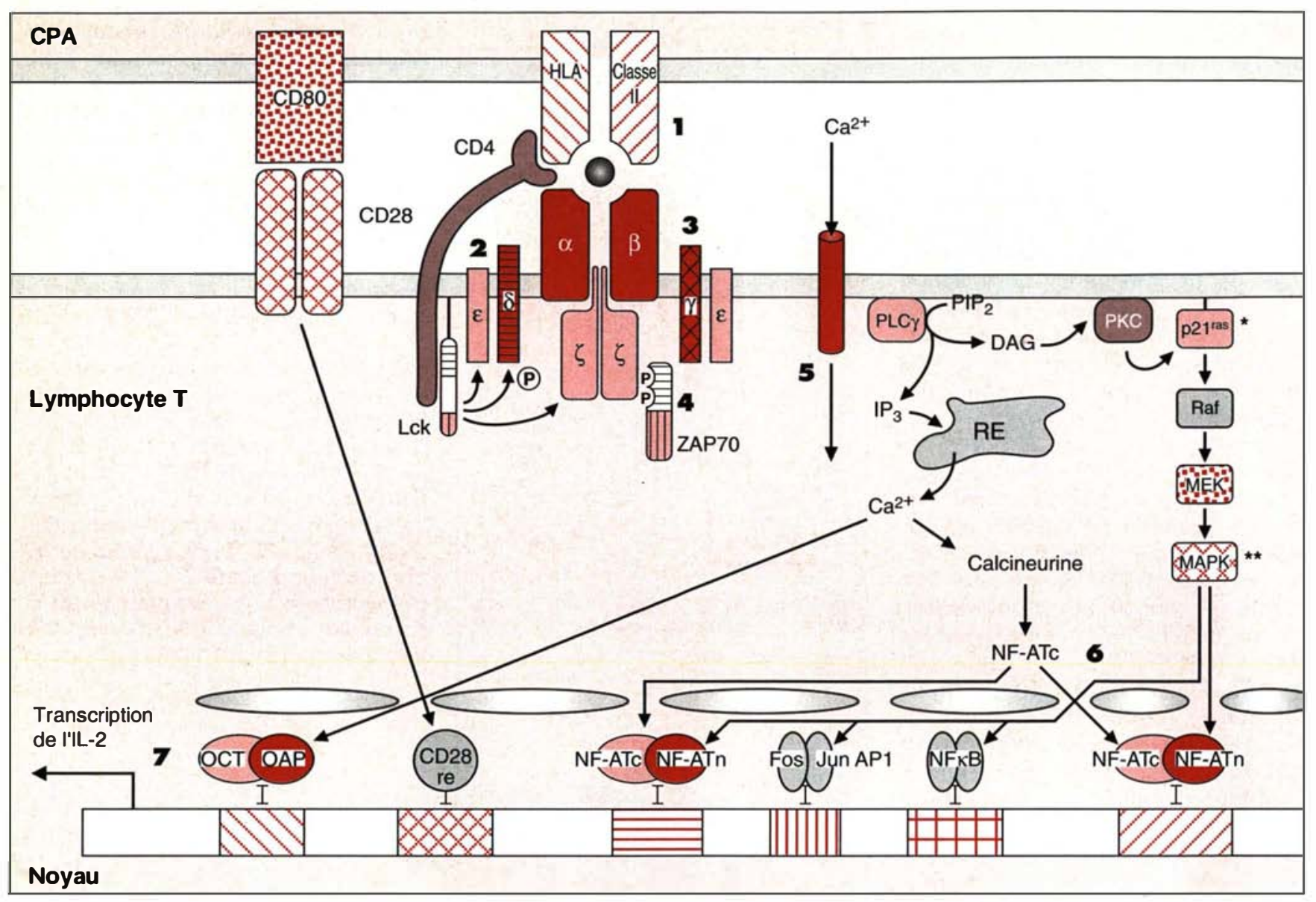

Figure 3. Événements précoces de l'activation des lymphocytes $T$ et déficits caractérisés. (1) défaut d'expression des

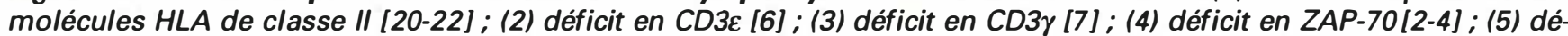
ficit en flux de Ca ${ }^{2+}[23,24] ;(6)$ déficit en NF-ATC $[25,26]$; (7) déficit de la production d'IL2 [27, 28]. *II existe aussi une voie d'activation de Ras dépendante de protéines tyrosine kinases. **a phosphorylation des facteurs de transcription, et en particulier de Jun, se fait également par une famille de kinases dont l'activation dépend de p2 $1^{r a s}$ et du $\mathrm{Ca}^{2+}$. CPA : cellule présentatrice de l'antigène ; PIP2 : phosphatidyl inositol bisphosphate ; DAG : diacylglycérol ; PKC : protéine kinase $C ; P L C \gamma$ : phospholipase $C ; R E$ : réticulum endoplasmique ; IP3 : inositol trisphosphate ; CD28re : CD28 response element ; OCT, OAP, NF-AT, Fos, Jun, NFKB : facteurs de transcription.

Les patients atteints présentent des infections récurrentes caractéristiques d'un déficit $T$. Les cellules $T$ périphériques de ces malades sont pourtant en nombre normal ; elles expriment de façon correcte le complexe CD3/TCR mais ne répondent pas à une stimulation passant par ce récepteur. Après activation par l'intermédiaire du CD3/TCR, on observe une phosphorylation très réduite des protéines cellulaires ainsi qu'une concentration intracytoplasmique de
$\mathrm{Ca}^{2+}$ anormalement élevée dans les cellules des patients, sans synthèse détectable d'IL2. En revanche, la prolifération induite par des agents court-circuitant les événements membranaires (phorbol esters et ionophore du calcium) reste intacte, montrant que seuls les événements précoces de l'activation par le CD3/TCR sont altérés.

Le fait le plus remarquable, chez les malades, est l'absence de lymphocytes $\mathrm{T} \mathrm{CD}^{+}$en périphérie où seuls les lymphocytes $\mathrm{T} \mathrm{CD} 4^{+}$sont présents mais non fonctionnels. Cette absence de cellules $\mathrm{T} \mathrm{CD}^{+}$en périphérie est due à un défaut de développement intrathymique des lymphocytes $\mathrm{T}$ : les thymocytes "doubles positifs" $\mathrm{CD}^{+} / \mathrm{CD}^{+}$sont présents dans le cortex thymique, mais seule la population "simple positive" $\mathrm{CD}^{+}$est détectée dans la médullaire.

Dans des conditions normales, les lymphocytes $\mathrm{T}$ murissent et quittent le thymus après avoir reçu un signal 
donné par le récepteur de l'antigène (phénomène appelé " sélection positive "). Chez les malades avec déficit en ZAP-70, il semble que ce soit précisément à l'étape de la sélection positive que réside le blocage.

Une question subsiste: pourquoi l'anomalie ne concerne-t-elle que la population $\mathrm{T} \mathrm{CD}^{+}$? Pour le moment, on ne connaît pas de voie d'activation spécifique du développement de l'une ou l'autre des populations de lymphocytes $\mathrm{T} \mathrm{CD}^{+}$ ou $\mathrm{T} \mathrm{CD8} 8^{+}$. Toutefois, une hypothèse a été émise. ZAP-70 et Syk sont exprimées dans toutes les populations thymiques $\left(\mathrm{CD}^{-} \mathrm{CD}^{-}, \mathrm{CD}^{+} \mathrm{CD}^{+}\right.$, $\left.\mathrm{CD}^{+} \mathrm{CD}^{-}, \mathrm{CD}^{-} \mathrm{CD}^{+}\right)$et pourraient toutes deux jouer un rôle dans la maturation thymique dépendante du CD3/TCR. Syk est exprimée dans les cellules thymiques à un niveau quatre fois supérieur à celui des cellules périphériques [19]. En l'absence de ZAP-70, Syk pourrait s'y substituer dans la sélection positive des thymocytes $\mathrm{T} \mathrm{CD}^{+}$, mais ne suffirait pas à compenser le déficit en ZAP-70 des cellules $\mathrm{T}$ mûres. Le développement préférentiel de la population $\mathrm{CD}^{+}$serait lié à une meilleure association de $\mathrm{p} 56^{\mathrm{lck}}$ avec la molécule CD4 qu'avec CD8. Ainsi, lors de la stimulation par le complexe CD3/TCR, un peptide associé aux molécules du CMH de classe II, interagissant avec CD4, induirait une meilleure activation de Lck et une meilleure phosphorylation de Syk qu'un peptide associé aux molécules du CMH de classe I interagissant avec CD8. Dans ce dernier cas, la présence de ZAP-70 serait indispensable, son absence entraînant l'absence de sélection positive des lymphocytes $\mathrm{T} \mathrm{CD8}^{+}$mûrs par des antigènes présentés dans le contexte du $\mathrm{CMH}$ de classe I. De plus, ZAP-70 serait également requise pour l'activation des lymphocytes $\mathrm{CD}^{+}$en périphérie.

On peut aussi envisager que la population $\mathrm{CD}^{+}$émerge en périphérie à la suite d'une maturation aberrante, le défaut de transmission des signaux passant par le complexe CD3/TCR observé dans les cellules mûres des patients ne serait alors pas seulement dû à l'absence de ZAP-70. Bien que toutes les questions concernant le rôle de ZAP-70 ne soient pas réso$m / s n^{\circ} 2$, vol. Il, fârier 95 lues, les observations faites sur les malades déficients en ZAP-70 attestent une fois encore de l'intérêt de telles études dans la compréhension des fonctions biologiques d'une protéine.

D'autres déficits d'activation des lymphocytes $\mathrm{T}$ ont été caractérisés au cours de ces dernières années. Ils sont schématisés sur la figure 3.

Le défaut d'expression des molécules d'histocompatibilité de classe II conduit à un défaut partiel de différenciation des lymphocytes $\mathrm{T} \mathrm{CD} 4$ et une absence d'activation de ces cellules [20]. Il est provoqué par plusieurs formes d'anomalies de régulation transcriptionnelle des gènes codant pour ces molécules. Ainsi, le déficit en transactivateurs CIITA [21] rend impossible la transcription de ces gènes.

Des déficits partiels et complets, res-

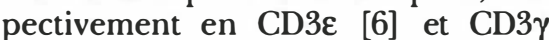
[7], inhibent partiellement l'activation des lymphocytes $T$. Il semble que la costimulation des lymphocytes $\mathrm{T}$, notamment par la molécule CD28, réduise partiellement les conséquences de ces déficits. Il est remarquable d'observer que l'absence de CD3 $\gamma$ réduit seulement l'expression CD3 d'un facteur deux. Nous avons récemment décrit un déficit profond de l'activation des lymphocytes $T$ dans deux familles. Le défaut correspond à un défaut d'entrée du calcium extracellulaire, alors que la libération du calcium stocké dans le réticulum endoplasmique sous l'effet de l'inositol tris-phosphate est conservé [22, 23]. Il pourrait s'agir d'un défaut d'un canal calcique (non encore cloné) ou d'un élément réglant la fonction de ce canal. Cette observation confirme l'importance d'une élévation substantielle et persistante de la concentration de calcium intracytoplasmique dans l'activation des lymphocytes $T$, en particulier dans l'induction de la transcription du gène codant pour l'IL2.

Chez un malade dont les lymphocytes $\mathrm{T}$ ne produisent pas d'IL2 ni d'lL4, une anomalie du composant cytosolique du facteur de transcription NF-AT a enfin été démontrée [24]. Ce résultat illustre l'importance du complexe NF-AT [25], dont l'acti- vité est réglée à la fois par la voie $\mathrm{PKC} / \mathrm{p} 21$ dépendante de Ras et par la voie dépendante du calcium et de la calcineurine [26].

Enfin, chez plusieurs patients, un défaut isolé de transcription du gène de l'IL2 a été mis en évidence sans que le mécanisme moléculaire responsable ait été caractérisé $[27,28]$. Il est évident que la description de nouveaux déficits immunitaires héréditaires qui perturbent l'activation lymphocytaire $\mathrm{T}$ contribuera encore dans un proche avenir à une meilleure compréhension des voies moléculaires complexes mises en jeu

\section{Claire Hivroz \\ Françoise Le Deist \\ Alain Fischer}

Inserm U. 429, Hôpital Necker-EnfantsMalades, 149, rue de Sèrres, 75015 Paris, France.

\section{TIRÉS À PART}

C. Hivroz.

\section{RÉFÉRENCES}

1. Fischer A. Severe combined immunodeficiencies. Immunodef Rev 1992 ; 3 : 83-100.

2. Arpaia E, Shahar M, Dadi H, Cohen A, Roifman CH. Defective T-cell receptor signalling and $\mathrm{CD}^{+}$thymic selection in humans lacking ZAP-70 kinase. Cell 1994 ; 76 : $1-20$.

3. Chan AC, Kadlecek T, Elder M, Filipovich $\mathrm{AH}$, Kuo WM, Iwashima $M$, Parslow $\mathrm{TR}$, Weiss A. ZAP-70 deficiency in an autosomal recessive form of severe combined immunodeficiency. Science $1994 ; 264$ : 1599-601.

4. Elder ME, Lin DL, Clever J, Chan AC Hope TJ, Weiss A, Parslow TG. Human severe combined immunodeficiency due to a defect in ZAP-70, a T-cell tyrosine kinase. Science 1994 ; 264 : 1596-9.

5. Soudais C, Lisowska-Grospierre B. Déficits immunitaires liés à un défaut d'expression du récepteur pour l'antigène. médecine/sciences 1993 ; $9: 764-7$.

6. Soudais C, de Villartay JP, Le Deist F, Fischer A, Lisowska-Grospierre $B$. Independent mutations of the human $C D 3-\varepsilon$ gene resulting in a T-cell receptor/CD3 complex immunodeficiency. Nature Genet $1993 ; 3$ : $77-81$. 
7. Arnaiz-Villena A, Timon $M$, Corell A, Perez-Aciego P, Martin-Villena JM, Regueiro JR. Primary immunodeficiency caused by mutations in the gene encoding the CD3 subunit of the T-lymphocyte receptor. $N$ Engl JMed 1992 ; 327 : 529-33.

8. Weiss A. T-cell antigen receptor signal transduction : a tale of tails and cytoplasmic protein-tyrosine kinases. Cell 1993; 73 : 209-12.

9. Weiss A, Littman DR. Signal transduction by lymphocyte antigen receptors. Cell 1994 ; 76 : 263-74.

10. Fagard R, Danielan S. Rôle des tyrosine protéine kinases dans l'activation des lymphocytes T. médecine/sciences $1989 ; 5: 554$ 60.

11. Molina TJ, Kishihara K, Siderovski DP, Van Ewijk W, Narendran A, Timms E, Wakeham A, Paige CJ, Hartmann KV, Veillette A, Davidson D, Mak TW. Profound block in thymocyte development in mice lacking $\mathrm{p} 56^{\mathrm{lck}}$. Nature $1992 ; 357: 161-4$.

12. Appleby MW, Gross JA, Cooke MP, Levin SD, Qian X, Perlmutter RM. Defective T-cell receptor signalling in mice lacking the thymic isoform of p59 $\mathrm{fm}$. Cell $1992 ; 70$ : 751-63.

13. Stein PL, Lee HM, Rich S, Soriano P. p59 $9^{\text {fyn }}$ mutant mice display differential signalling in thymocytes and peripheral $\mathrm{T}$ cells. Cell 1992; 70 : 741-50.

14. Chan AC, Iwashima M, Tuck CW, Weiss A. A 70 Kd protein tyrosine kinase that associates with the T-cell receptor $\zeta$ chain. Cell $1992 ; 71$ : 649-62.
15. Chardin P. Domaines SH2 et SH3: un nouveau paradigme pour la transmission du signal. medecine/sciences $1994 ; 10: 709-12$.

16. Chan AC, Irving BA, Fraser JD, Weiss A The $\zeta$ chain is associated with a tyrosine $\mathrm{ki}$ nase and upon T-cell antigen receptor stimulation associates with ZAP-70, a $70 \mathrm{kDa}$ tyrosine phosphoprotein. Proc Natl Acad Sa USA 1991 ; 88 : $9166-70$

17. Straus DB, Weiss A. The CD3 chains of the T-cell antigen receptor associate with the ZAP-70 tyrosine kinase and are tyrosine phosphorylated after receptor stimulation. $J$ Exp Med 1993 ; 178 : 1523-30.

18. Iwashima M, Irving BA, Van Oers NSC Chan AC, Weiss A. Sequential interactions of the TCR with two distinct cytoplasmic ty rosine kinases. Science 1994 ; 263 : 1136.

19. Chan AC, Van Oers NSC, Tran A, Turka L, Law CL, Ryan JC, Clark EA, Weiss A. Differential expression of ZAP-70 and Syk protein tyrosine kinases, and the role of this family of protein tyrosine kinases in TCR signaling. JImmunol 1994 ; 152 : 4758-66.

20. Griscelli C, Lisowska-Grospierre B, Mach B. Combined immunodeficiency with defective expression in MHC class II genes. New concepts in immunodeficiency diseases. New York : John Wiley, 1993 : 177-90.

21. Steimle V, Siegrist CA, Mottet A, Lisowska-Grospierre B, Mach B. Regulation of MHC class II expression by interferon $\gamma$ is mediated by the transactivator gene CIITA. Science 1994 ; 265 : 106-9.
22. Le Deist F, Hivroz $C$, Partiseti $M$, Thomas C, Buc HA, Oleastro M, Belohradsky B Choquet D, Fischer A. A primary T-cell immunodeficiency associated with defective transmembrane calcium influx. Blood 1995 (sous presse).

23. Partiseti $M$, Le Deist F, Hivroz C, Fischer A, Korn H, Choquet D. The calcium current activated by T-cell receptor and store depletion in human lymphocytes is absent in a primary immunodeficiency. $J$ Biol Chem 1995 (sous presse).

24. Castigli E, Pawha R, Good RA, Geha RS, Chatila TA. Molecular basis of a multiple lymphokine deficiency in a patient with severe combined immunodeficiency. Proc Nall Acad Sci USA 1993 ; 90 : 4728-32.

25. Israēl A. La vérité sur NF-AT. médecine/sciences $1994 ; 10: 894-5$.

26. Grabtree GR, Clipstone NA. Signal transmission between the plasma membrane and nucleus of T lymphocytes. Annu Rev Biochem 1994 ; 63 : 1045-83.

27. Weinberg K, Parkman R. Severe combined immunodeficiency due to a specific defect in the production of interleukin $2 . N$ Engl J Med 1990 ; 327 : 1718-23.

28. Disanto IP, Keever CA, Small TN, Nichols GL, O'Reilly RJ, Flomenberg N. Absence of interleukin 2 production in a severe combined immunodeficiency disease syndrome with T cells. $J \operatorname{Exp} M e d 1990 ; 171$ : 1697-704. 